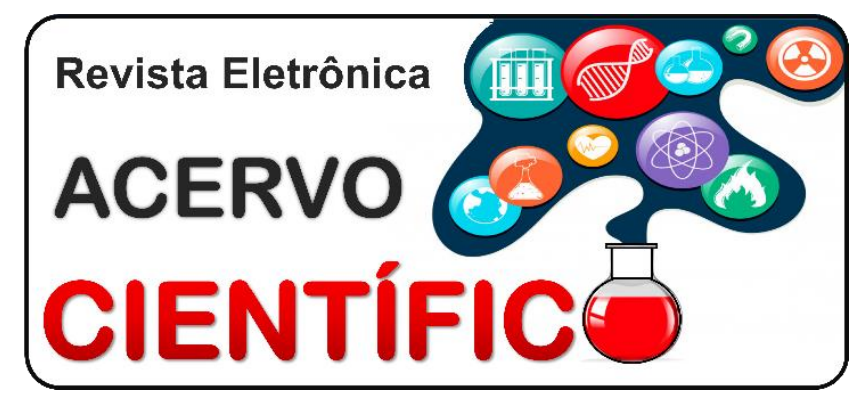

REVISÃO BIBLIOGRÁFICA

Recebido em: 11/2020

Aceito em: $11 / 2020$

Publicado em: 12/2020

\title{
Revisão sistemática sobre o uso da flibanserina no desejo sexual hipoativo em mulheres na pré menopausa
}

\author{
Systematic review about the use of flibanserin in hypoactive sexual desire in women pre \\ menopausing
}

\section{Revisión sistemática sobre el uso de flibanserina en el deseo sexual hipoactivo en mujeres premenopáusicas}

Marina Leite Oliveira ${ }^{1 *}$, Julia Gonçalves Faleiros Cardoso ${ }^{1}$, lara Chaves de Souza ${ }^{1}$, Letícia Pontes Reis ${ }^{1}$, Isadora Ávila Chagas da Silva ${ }^{1}$, Gabriela Dias Macedo ${ }^{1}$, Sanzio Rafael Faria de Vasconcelos ${ }^{1}$, Ana Luísa Melgaço Almeida², Ana Luiza Ledro Nagem¹, Maria Aparecida Turci².

\begin{abstract}
Resumo: O objetivo do presente artigo foi avaliar o uso da Flibanserina em comparação ao placebo na melhora do desejo sexual hipoativo (DSH) em mulheres na pré-menopausa. Realizou-se um Protocolo de Revisão Sistemática com busca nas bases de dados Cochrane, Pubmed e Biblioteca Virtual do Ministério da Saúde (BVSaúde). Os artigos foram selecionados por uma dupla de revisores independentes, considerando critérios de inclusão e de exclusão pré-definidos. Esta Revisão Sistemática contém 8 ensaios clínicos randomizados (ECR), derivados de 474 artigos. Os estudos analisaram a capacidade da Flibanserina em aumentar a libido das pacientes diagnosticadas com DSH, excluídas outras causas. Os desfechos demonstraram eficácia do medicamento utilizado na posologia de $50 \mathrm{mg}$ ou $100 \mathrm{mg}$, por, no mínimo, 24 semanas, apesar de efeitos colaterais. Pode-se concluir que uso da Flibanserina no tratamento do DSH, comparado ao placebo, foi eficaz, se usado por 24 semanas, nas doses de $50 \mathrm{mg}$ ou $100 \mathrm{mg}$.
\end{abstract}

Palavras-chave: Flibanserina, Desejo sexual, Hipoativo.

\begin{abstract}
The objective of this article was evaluate the use of flibanserin in comparison to the placebo in increasing sexual desire in women pre menopausing. A systematic review protocol was done by means of a research in the data basis Cochrane, Pubmed and BVSaúde. The papers were selected by two independent revisors, considering pre-definite inclusion and exclusion criteria. This systematic review contains 8 randomized clinical trials, derived from 474 articles. The studies analyzed the capacity of flibanserin to increase libido in patients diagnosed with hypoactive sexual desire, excluding other causes. The outcome demonstrated efficacy of the drug when administrated in a dosage between $50 \mathrm{mg}$ and $100 \mathrm{mg}$, during at least 24 weeks, despite all the side effects. It is possible to conclude that use of flibanserin in the treatment of post menopause women with hypoactive sexual desire, compared to the placebo, was efficient, if used for a minimum of 24 weeks, in the dosage between $50 \mathrm{mg}$ and $100 \mathrm{mg}$.
\end{abstract}

Key words: Flibanserin, Sexual desire, Hypoactive.

\footnotetext{
1 Universidade José do Rosário Vellano (UNIFENAS), Belo Horizonte - MG.

*E-mail: marina leite12@hotmail.com

2 Universidade Federal de Minas Gerais (UFMG), Belo Horizonte - MG.
} 
Resumen: El objetivo del artigo fue evaluar el uso de la Flibanserina en comparación al placebo en la mejora del deseo sexual hipo activo (DSH) en mujeres en la peri menopausia. Se ha hecho un Protocolo de Revisión Sistemática con búsqueda en las bases de datos Cochrane, Pubmed y Biblioteca Virtual del Ministerio de la Salud (BVSaúde). Los artículos han sido seleccionados por una pareja de revisores independientes, considerando criterios de inclusión y exclusión predefinidos. Resultados: Esta Revisión Sistemática contiene 8 ensayos clínicos aleatorizados (ECA), derivados de 474 artículos. Los estudios han analizado la capacidad de la Flibanserina en aumentar la libido de las pacientes diagnosticadas con DSH, excluidas otras causas. Los resultados han demostrado eficacia del medicamento utilizado en la posología de $50 \mathrm{mg}$ o $100 \mathrm{mg}$, por lo menos 24 semanas, a pesar de los efectos adversos. Se puede concluir que el uso de la Flibanserina en el tratamiento del DSH, comparado al placebo, ha sido eficaz, si usado por 24 semanas, en las dosis de $50 \mathrm{mg}$ o $100 \mathrm{mg}$.

Palabras-clave: Flibanserina, Deseo sexual, Hipo activo.

\section{INTRODUÇÃO}

O desejo sexual hipoativo é uma queixa comum de mulheres na pré-menopausa. Trata-se de uma condição que afeta cerca de $5,4 \%$ a $13,6 \%$ das mulheres em tal fase e é caracterizada por ausência de fantasias sexuais e de desejo pela prática, ocasionando um sofrimento pessoal acentuado ou mesmo uma dificuldade interpessoal nos relacionamentos. Essas repercussões atingem um pico em mulheres de 45 a 64 anos. Para o seu correto diagnóstico, deve-se excluir distúrbios psiquiátricos, uso de medicamentos ou condição médica que possam resultar em qualquer desordem do desejo sexual. Os tratamentos mais disponíveis se baseiam na abordagem dos sintomas específicos, uma vez que esse distúrbio possui etiologia multifatorial. No entanto ainda carece de tratamento que aborde o transtorno como um todo (HOUMAN J, et al., 2012).

De acordo com a Organização Mundial da Saúde (OMS), saúde sexual consiste em um estado psicológico, emocional, social, sustentado pelos direitos humanos em relação a sexualidade, que promove vida sexual segura, autônoma e satisfatória. Com base em sua importância e devido a alta prevalência de queixas neste âmbito, as últimas décadas foram marcadas por um avanço na medicina sexual, com o intuito de fornecer soluções para o atendimento de homens e mulheres com problemas sexuais. Acredita-se que a fisiopatologia da desordem do desejo sexual hipoativo provém de uma deficiência relativa na atividade dos neurotransmissores dopaminérgicos e noradrenérgicos, que exercem efeitos excitatórios no sistema nervoso central, e um relativo excesso de atividade serotoninérgica, que exerce um efeito inibitório no córtex préfrontal. A modulação desses fatores pode ajudar a restaurar o equilíbrio necessário para uma resposta sexual saudável (NAPPI RE, et al., 2015).

Estudos epidemiológicos estimaram que uma a cada dez mulheres relata diminuição do desejo sexual associado a angústia. No entanto, o desejo sexual hipoativo entre a população do sexo feminino permanece inúmeras vezes sem diagnóstico e tratamento adequados. A Flibanserina é um agonista 5-HT1A pós-sináptico / 5- Antagonista HT2A. Quando investigado como uma opção para tratamento para transtorno depressivo maior (MDD), foi considerado um antidepressivo ineficaz, mas, durante os estudos, foi observado que esse medicamento aumenta o desejo sexual em mulheres com MDD que tinham desejo sexual hipoativo no início do estudo. Isso forneceu uma justificativa para investigação da Flibanserina como uma nova opção no tratamento do desejo sexual hipoativo em mulheres na pré-menopausa (DEROGATIS LR, et al., 2012).

Diversos estudos acerca das possíveis interferências benéficas no período da pré-menopausa foram realizados e culminaram na liberação, em agosto de 2015, do fármaco Addyi® da empresa Sprout Pharmaceuticals, cujo princípio ativo é a Flibanserina (CLAYTON AH, 2010).

Atualmente, sabe-se que a Flibanserina é um medicamento que provoca aumento do desejo sexual, uma vez que atua como um agonista da atividade dopaminérgica e noradrenérgica, e como um antagonista na via serotoninérgica. Outro mecanismo de ação que tal fármaco possui é a metabolização pelas enzimas do citocromo P450 e uma meia-vida de, aproximadamente, onze horas, sendo contraindicado em pacientes com 
insuficiência hepática, visto que tal moléstia aumenta a exposição e a meia-vida do medicamento. Além disso, a Flibanserina é contra indicada em pacientes que fazem o uso excessivo de bebidas alcoólicas, pois, essa combinação aumenta significativamente o risco de hipotensão e síncope. Seu uso surge nesse contexto a fim de garantir uma melhora da qualidade de vida dessas mulheres que se encontram no período pré menopausa com problemas sexuais (DEROGATIS LR, et al., 2012).

Como o desejo sexual feminino ainda é um tema pouco abordado e a Flibanserina é pouco conhecida, foi optada pela realização de uma revisão sistemática dos estudos já existentes, sendo possível se obter uma maior compreensão dos efeitos deste fármaco sobre o bem estar físico e psicológico das mulheres. Assim, o objetivo dessa revisão de literatura é avaliar os efeitos da Flibanserina quando usada em mulheres na prémenopausa para o tratamento de desejo sexual hipoativo.

\section{MÉTODOS}

Este artigo foi realizado com base no Protocolo de Revisão Sistemática, no qual todos os detalhes do método do estudo foram citados, além do título e de uma introdução sobre o tema abordado, e por fim, a afirmação sobre a importância desse trabalho tendo em vista sua prevalência na atualidade.

Realizou-se uma revisão sistemática de literatura sobre a Flibanserina e o aumento do desejo sexual em mulheres na pré-menopausa. Os artigos foram buscados nas bases de dados online Cochrane, Pubmed e Biblioteca Virtual do Ministério da Saúde (BVSaúde).

Para estratégia de busca foram utilizadas as palavras-chave: Flibanserin OR Flibanserina AND Prémenopausa OR Premenopause AND desejo sexual OR sexual desire AND Hypoactive sexual desire disorder. Como descritores, foram usados: "Flibanserin", encontrado na busca de descritores do Pubmed; "Libido" e "Pré-Menopausa", encontrados na busca na Biblioteca Virtual em Saúde.

Cada plataforma de base de dados teve seus artigos selecionados por uma dupla de revisores, feitas de maneira independente. Dessa forma, na primeira etapa da seleção de artigos, foi feita a triagem por meio da leitura de título e resumo de acordo com os critérios de inclusão pré-definidos. São esses critérios: Ensaios clínicos publicados em um período de até 10 anos e mulheres na pré-menopausa com desejo sexual hipoativo sem causa específica.

Após esse processo, foram excluídos os artigos duplicados. A partir disso, foi realizada a segunda etapa de busca: Leitura do texto completo por uma dupla de revisores, de modo independente e a exclusão de artigos de acordo com os critérios de exclusão.

São esses critérios: Mulheres na pós-menopausa, mulheres em uso de álcool, drogas e/ou outros medicamentos de uso contínuo, uso da Flibanserina não relacionado ao desejo sexual hipoativo, desejo sexual de causa especificada ou por doença de base, artigos de revisão de literatura, adendos, editoriais, opiniões de especialistas e artigos indisponíveis, como podemos ver na Figura 1. 
Figura 1 - Fluxograma de extração de dados.

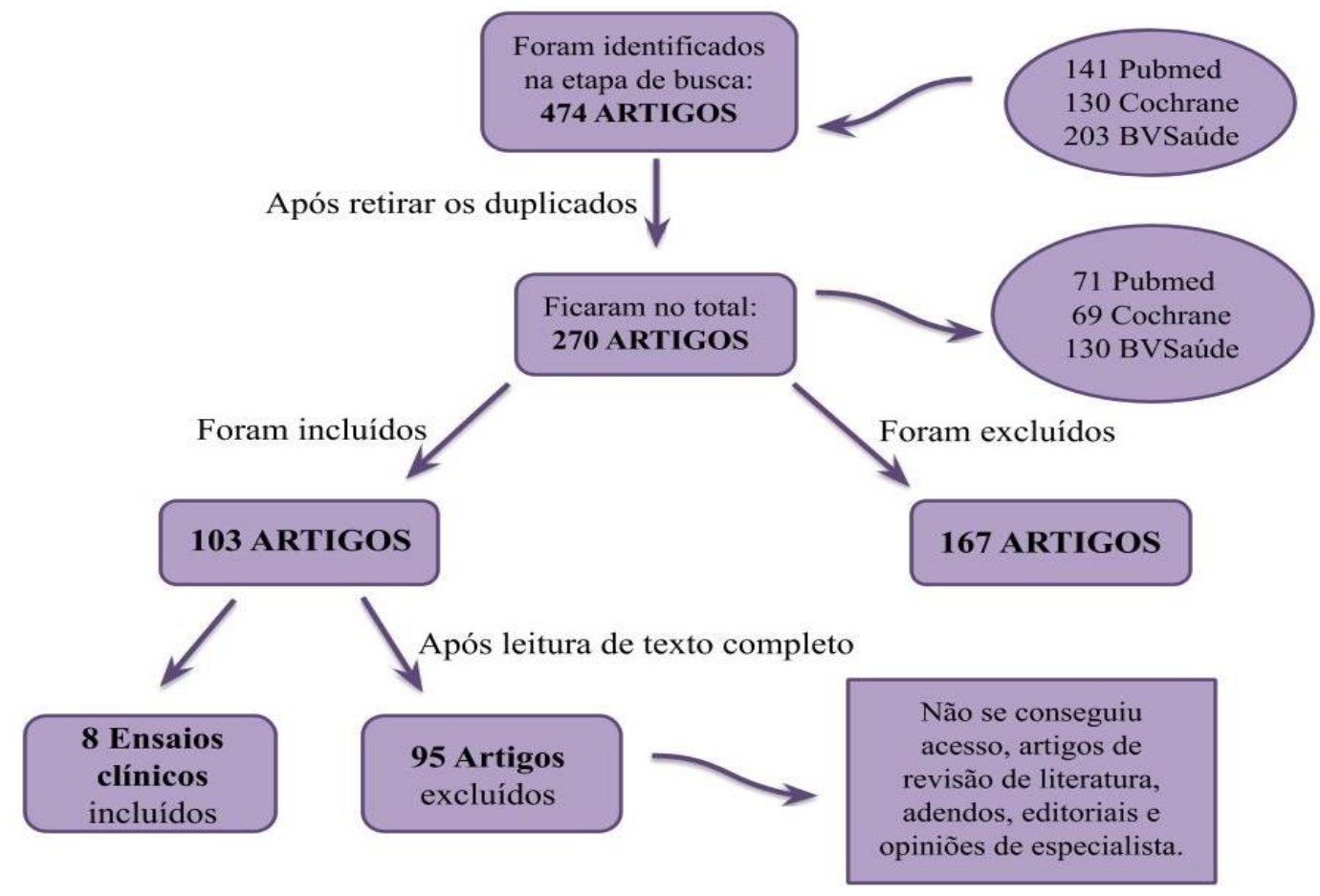

Fonte: OLIVEIRA ML, et al., 2020.

\section{RESULTADOS}

A presente revisão sistemática foi composta por 8 estudos ensaio clínico, derivados de 474 artigos inicialmente encontrados, sendo esses 141 da base Pubmed, 130 artigos da base Cochrane e 203 da plataforma BVSaude. Foram então selecionados 270 estudos após retirar os artigos duplicados (71 artigos da base Pubmed, 69 artigos da Cochrane e 130 artigos BVSaude).

Depois da leitura de títulos e resumos, foram selecionados 103 artigos, que foram lidos completamente. Dessa forma, 95 foram excluídos com base nos critérios anteriormente citados, além de artigos que não se conseguiu acesso, artigos de revisão de literatura, adendos, editoriais e opiniões de especialistas, resultando nos 8 ensaios clínicos utilizados para a síntese dos resultados. Os artigos analisados e utilizados para a construção desta revisão sistemática apresentam intervalo de publicação de até dez anos atrás. Todos os estudos foram sediados nos Estados Unidos e têm como objetivo principal analisar se a Flibanserina é capaz de aumentar a libido de mulheres que estejam no período da pré-menopausa e tenham o diagnóstico de desejo sexual hipoativo.

O número de pacientes avaliadas em cada estudo variou de 60 a 2465, sendo que, todas apresentavam distúrbio sexual, como a diminuição da libido. Os esquemas terapêuticos foram variados entre $25 \mathrm{mg}, 50 \mathrm{mg}$ e $100 \mathrm{mg}$ e utilizados de 20 a 52 semanas. Dentre os onze ensaios clínicos avaliados, 10 comparam o uso da Flibanserina em relação ao placebo, enquanto apenas 1 usa como contraponto a psicoterapia sexual (Quadro 1).

Os efeitos das intervenções realizadas foram, em sua maioria, mensurados por meio da aplicação de questionários, como por exemplo, o Female Sexual Function Index (FSFI), quantidade de relações sexuais satisfatórias em 28 dias e uso de diários feitos pelas participantes. Apenas um estudo afirma a presença de duplo cegamento, aumentando a confiabilidade de suas informações, enquanto outro relata não ter sido feito em sua metodologia (Quadro 1).

Os resultados dos estudos demonstraram que o tratamento foi eficaz quando o medicamento foi usado na posologia de $50 \mathrm{mg}$ ou $100 \mathrm{mg}$, por, no mínimo, 24 semanas apesar dos possíveis efeitos colaterais relacionados ao seu uso, como sonolência, fadiga, náuseas, vômitos, tontura, entre outros (Quadro 1). 
Quadro 1 - Tabela de resultados dos Ensaios Clínicos analisados.

\begin{tabular}{|c|c|c|c|}
\hline Ensaio Clínico & Método & Dose/Tempo de tratamento & Desfecho \\
\hline $\begin{array}{l}\text { Eficácia da Flibanserina em mulheres com } \\
\text { transtorno do desejo sexual hipoativo: } \\
\text { Resultados do ensaio clínico BEGONIA. } \\
\text { (KATZ M, et al., 2013) }\end{array}$ & $\begin{array}{l}\text { ECR com } 1087 \text { mulheres, sendo que, } \\
542 \text { dessas fizeram o uso de } \\
\text { Flibanserina e } 545 \text { placebo por } 24 \\
\text { semanas. Os efeitos foram avaliados } \\
\text { através de questionários }\end{array}$ & 100mg/dia por 24 semanas. & $\begin{array}{l}\text { Mostrou a eficácia da Flibanserina no } \\
\text { tratamento do desejo sexual hipoativo } \\
\text { em comparação com o placebo, mas } \\
\text { não informou se houve cegamento. }\end{array}$ \\
\hline $\begin{array}{l}\text { Tratamento do transtorno de desejo } \\
\text { sexual hipoativo em mulheres na pré- } \\
\text { menopausa: Eficácia da Flibanserina no } \\
\text { estudo VIOLETA. } \\
\text { (DEROGATIS LR, et al., 2012) }\end{array}$ & $\begin{array}{c}\text { ECR com } 590 \text { mulheres, sendo que, } \\
295 \text { utilizaram placebo e } 295 \text { fizeram } \\
\text { o uso de Flibanserina por } 24 \\
\text { semanas. Os efeitos foram avaliados } \\
\text { por meio de questionários }\end{array}$ & $\begin{array}{c}100 \mathrm{mg} / \mathrm{dia} \text { ou } 50 \mathrm{mg} / \mathrm{dia} \text { por } 24 \\
\text { semanas. }\end{array}$ & $\begin{array}{l}\text { O uso da Flibanserina foi indicado pelo } \\
\text { estudo com finalidade de melhorar o } \\
\text { desejo sexual hipoativo nas doses de } \\
\text { 100mg ou } 50 \mathrm{mg} \text { por } 24 \text { semanas, } \\
\text { mostrando a eficácia do medicamento } \\
\text { quando usado dessa forma. }\end{array}$ \\
\hline $\begin{array}{l}\text { Tratamento do transtorno do desejo } \\
\text { sexual hipoativo em mulheres na pré- } \\
\text { menopausa: Eficacia da Flibanserina no } \\
\text { estudo DAISY. } \\
\text { (THORP J, et al., 2012) }\end{array}$ & $\begin{array}{l}\text { ECR com } 793 \text { mulheres, sendo que, } \\
398 \text { utilizaram placebo e } 395 \text { fizeram } \\
\text { o uso de Flibanserina alternando a } \\
\text { dosagem do medicamento por } 24 \\
\text { semanas. Os efeitos foram avaliados } \\
\text { por meio de questionários }\end{array}$ & $\begin{array}{c}\text { 25mg BID, } 50 \mathrm{mg} \text { BID e } 100 \mathrm{mg} \text { MID } \\
\text { por } 24 \text { semanas. }\end{array}$ & $\begin{array}{c}\text { Depois do uso das ferramentas } \\
\text { indicadas, o estudo comprovou que o } \\
\text { uso da Flibanserina é seguro, apesar } \\
\text { dos efeitos colaterais relatados e que o } \\
\text { uso de } 100 \mathrm{mg} \text { se mostrou adequado } \\
\text { fazendo com que os efeitos esperados } \\
\text { fossem encontrados (aumento do } \\
\text { desejo sexual). }\end{array}$ \\
\hline $\begin{array}{l}\text { Eficácia e segurança continuados da } \\
\text { Flibanserina em mulheres na pré- } \\
\text { menopausa com transtorno do desejo } \\
\text { sexual hipoativo (TDSH): Resultados de } \\
\text { um ensaio clínico randomizado. } \\
\text { (GOLDFISCHER ER, et al., 2011) }\end{array}$ & $\begin{array}{l}\text { ECR com } 738 \text { mulheres, sendo que, } \\
\text { nas primeiras } 24 \text { semanas todas as } \\
\text { participantes utilizaram Flibanserina } \\
\text { de } 50 \text { ou } 100 \mathrm{mg} \text {. Depois da } 24^{\underline{a}} \\
\text { semana as mulheres que tiveram } \\
\text { benefícios com o medicamento foram } \\
\text { redivididas, sendo que } 163 \text { utilizaram } \\
\text { Flibanserin e } 170 \text { placebo. Os } \\
\text { resultados foram avaliados por } \\
\text { questionários }\end{array}$ & $\begin{array}{l}50 \mathrm{mg} / \mathrm{dia} \text { ou } 100 \mathrm{mg} / \mathrm{dia} \text { por } 48 \\
\text { semanas. }\end{array}$ & $\begin{array}{l}\text { Flibanserina foi superior ao placebo, } \\
\text { quando usada por } 24 \text { semanas, na } \\
\text { melhora do desejo sexual hipoativo. }\end{array}$ \\
\hline
\end{tabular}




\begin{tabular}{|c|c|c|c|}
\hline Ensaio Clínico & Método & Dose/Tempo de tratamento & Desfecho \\
\hline $\begin{array}{l}\text { Estudo não-cego sobre o uso da } \\
\text { flibanserina em mulheres com transtorno } \\
\text { do desejo sexual hipoativo. } \\
\text { (JAYNE C, et al., 2012) }\end{array}$ & $\begin{array}{c}\text { ECR de } 52 \text { semanas que utilizou } \\
\text { doses flexíveis de Flibanserina MID } \\
\text { ou BID e os efeitos foram } \\
\text { comparados com placebo. }\end{array}$ & $\begin{array}{c}50 \mathrm{mg} \text { ou } 100 \mathrm{mg} \text { MID; } 25 \mathrm{mg} \text { ou } 50 \mathrm{mg} \\
\text { BID por } 52 \text { semanas. }\end{array}$ & $\begin{array}{c}\text { Medicamento apresentou muitos } \\
\text { efeitos colaterais, porém, foi eficaz } \\
\text { quando usado em 100mg durante } 4 \\
\text { semanas. }\end{array}$ \\
\hline $\begin{array}{l}\text { Uso da flibanserina para o transtorno do } \\
\text { desejo sexual hipoativo na pré- } \\
\text { menopausa: Análise conjunta de ensaios } \\
\text { clínicos. } \\
\text { (SIMON JA, et al., 2019) }\end{array}$ & $\begin{array}{c}\text { ECM com } 2.465 \text { mulheres, sendo } \\
\text { que } 1227 \text { usaram flibanserina, e } \\
1238 \text { usaram placebo. Os efeitos } \\
\text { foram avaliados através de uma } \\
\text { escala. }\end{array}$ & $100 \mathrm{mg} /$ dia por 24 semanas & $\begin{array}{c}\text { A flibanserina melhorou o desejo } \\
\text { sexual e reduziu o sofrimento sexual } \\
\text { associado com desejo sexual hipoativo. } \\
\text { Estudo não define se houve } \\
\text { cegamento. }\end{array}$ \\
\hline $\begin{array}{l}\text { Flibanserina em mulheres com transtorno } \\
\text { do desejo sexual hipoativo na pré- } \\
\text { menopausa: Uma análise de ensaios } \\
\text { clínicos randomizados. } \\
\text { (SIMON JA, et al., 2018) }\end{array}$ & $\begin{array}{c}\text { ECR com } 2022 \text { mulheres, sendo que, } \\
985 \text { fizeram o uso de Flibanserina e } \\
1037 \text { usaram placebo por } 24 \\
\text { semanas. Os efeitos foram avaliados } \\
\text { através de questionários }\end{array}$ & $100 \mathrm{mg} /$ dia por 24 semanas & $\begin{array}{c}\text { O estudo mostrou alta resposta ao } \\
\text { tratamento, com tempo médio de } \\
\text { aproximadamente } 2 \text { meses para ter os } \\
\text { efeitos desejados. É um estudo duplo } \\
\text { cego. }\end{array}$ \\
\hline $\begin{array}{l}\text { Efeito da Flibanserina na função sexual: } \\
\text { Uma análise dos índices de domínios da } \\
\text { função sexual feminina. } \\
\text { (KRYCHMAN ML, et al., 2017) }\end{array}$ & $\begin{array}{l}\text { ECR com } 2368 \text { mulheres, sendo que, } \\
1165 \text { dessas fizeram o uso de } \\
\text { Flibanserina e } 1204 \text { usaram placebo } \\
\text { por } 24 \text { semanas. Os efeitos foram } \\
\text { avaliados através de questionários }\end{array}$ & $100 \mathrm{mg} /$ dia por 24 semanas & $\begin{array}{l}\text { O tratamento com flibanserina resultou } \\
\text { em melhora significativa do desejo } \\
\text { sexual. É um estudo duplo cego. }\end{array}$ \\
\hline
\end{tabular}

Fonte: OLIVEIRA ML, et al., 2020. 


\section{DISCUSSÃO}

Para a realização do presente trabalho, optou-se pela escolha de ensaios clínicos randomizados, devido à maior confiabilidade desse tipo de estudo para o desenvolvimento de uma revisão sistemática. Os trabalhos demonstraram que a Flibanserina é um importante recurso terapêutico para o tratamento do desejo sexual hipoativo em mulheres na pré-menopausa, pois, essa é uma condição que é muito prevalente, porém, pouco discutida e com poucas opções de tratamento (SIMON JA, et al., 2018; KRYCHMAN ML, et al., 2017).

O transtorno do desejo sexual hipoativo "Hypoactive sexual desire disorder" (HSDD) é o problema de saúde sexual mais prevalente nas mulheres e é definido como a deficiência persistente ou recorrente ou a ausência de fantasias sexuais e desejo de atividade sexual, associado à angústia acentuada ou dificuldade interpessoal que não é explicada por uma condição médica ou psiquiátrica geral. Esse transtorno pode ser primário ou secundário, vitalício ou adquirido (GOLDSTEIN IR, et al., 2017; KRYCHMAN ML, et al., 2017).

A patogênese do HSDD permanece caracterizada de forma incompleta quanto as alterações biológicas. Clinicamente, nenhuma diferença hormonal identificável caracteriza as mulheres na pré-menopausa com HSDD. Embora alguns estudos relacionem baixos níveis de testosterona e diminuição dos níveis de estradiol com HSDD, não há nenhum nível hormonal que preveja essa alteração no desejo sexual. Assim, embora os corticosteroides ovarianos possam modular o desejo sexual das mulheres, seu papel exato na HSDD permanece obscuro (GOLDSTEIN IR, et al., 2017; THORP J, et al., 2012; SIMON JA, et al., 2019).

A definição ampliada de HSDD pode incluir qualquer um dos seguintes: (1) falta de motivação para atividade sexual, manifestada por desejo espontâneo reduzido ou ausente (pensamentos ou fantasias sexuais); desejo responsivo reduzido ou ausente a sugestões eróticas e estimulação; incapacidade de manter desejo ou interesse através de atividade sexual ou (2) perda de desejo de iniciar ou participar de atividade sexual, incluindo respostas comportamentais como evitar situações que poderiam levar à atividade sexual, isto é, não secundárias a distúrbios de dor sexual. Além disso, há a presença de sofrimento pessoal clinicamente significativo que inclui frustração, tristeza, sentimento de incompetência ou preocupação (GOLDSTEIN IR, et al., 2017; DEROGATIS LR, et al., 2012).

Descobriu-se que as mulheres com HSDD têm uma imagem corporal comprometida, redução da autoconfiança e da autovalorização e sentem-se menos conectadas a seus parceiros. Os gastos totais com cuidados de saúde em comparação com uma coorte de pacientes de controle foram maiores para mulheres com HSDD, incluindo consultas ambulatoriais, uso de medicamentos prescritos e outros serviços médicos, incluindo procedimentos de radiologia, laboratoriais e consultas ambulatoriais. As comorbidades presentes nessas mulheres incluem depressão e fadiga, assim como diabetes e dor nas costas (GOLDSTEIN IR, et al., 2017; DEROGATIS LR, et al., 2012).

Estudos epidemiológicos mostraram que mulheres com baixo desejo sexual possuem menor satisfação física e emocional com parceiros sexuais e felicidade geral reduzida em relação à mulheres sem problemas sexuais. $O$ que pode acarretar redução na qualidade de vida, distúrbios emocionais e insatisfação no relacionamento (GOLDSTEIN IR, et al., 2017; SIMON JA, et al., 2018; THORP J, et al., 2012).

Posteriormente, outros estudos encontraram associações entre desejo sexual baixo e menor qualidade de vida relacionada à saúde, bem como fatores psicossociais, como insatisfação com a vida sexual, parceiro ou casamento e estados emocionais negativos, incluindo frustração, desesperança, raiva, baixa auto-estima e perda de feminilidade. Houve também associação marcada com depressão e ansiedade em mulheres com desejo sexual hipoativo. Quando subgrupos foram ainda caracterizados, mulheres com um cônjuge ou parceiro atual eram mais propensos a experimentar sofrimento concomitante com baixo desejo sexual do que mulheres sem parceiros (GOLDSTEIN IR, et al., 2017; JAYNE C, et al., 2012).

Através de estudos de tomografia por emissão de pósitrons de voluntários, analisados durante vídeos eróticos, observa-se que mulheres com HSDD tem ativação mais fraca do córtex cerebral no hemisfério direito, e menos desativação no hemisfério esquerdo, em comparação com mulheres sem alteração do desejo sexual. É possível que a ativação mais fraca do lado direito possa representar uma resposta muda aos estímulos sexuais, enquanto a incapacidade de desativar o processamento analítico de ordem superior no 
lado esquerdo pode perpetuar as vias neurais inibitórias. Mecanicamente, a neuroplasticidade baseada na experiência pode explicar como várias modalidades de tratamento psicológico e médico podem beneficiar um paciente com HSDD ao modular potencialmente a inibição sexual subjacente e os mecanismos de excitação no cérebro (GOLDSTEIN IR, et al., 2017).

A excitação sexual envolve as ações da dopamina do cérebro, melanocortina, ocitocina, vasopressina e norepinefrina. Esses neurotransmissores coordenam as vias do hipotálamo, do sistema límbico e do córtex para processar e responder aos estímulos. A inibição sexual envolve os sistemas opióides, serotoninérgicos e endocanabinóides no cérebro que são ativados normalmente durante a refratariedade sexual ou como uma função de aversão primária ou evitação secundária. Esses sistemas inibitórios reduzem a capacidade dos sistemas excitatórios de serem ativados. Os medicamentos para o tratamento de HSDD devem seletivamente ativar estas vias estimulatórias ou reduzir as vias inibitórias, para que sejam efetivos (GOLDSTEIN IR, et al., 2017; DEROGATIS LR, et al., 2012).

A Flibanserina é um fármaco não hormonal de ação central agonista pós-sináptico do receptor de serotonina $1 \mathrm{~A}$ e um antagonista do receptor de serotonina $2 \mathrm{~A}$, que resulta em diminuição da atividade da serotonina e aumento de atividade da dopamina e norepinefrina. Devido à sua recente aprovação, a presente revisão tem sua importância ao analisar os possíveis desfechos do uso deste medicamento (GOLDSTEIN IR, et al., 2017; JAYNE C, et al., 2012; SIMON JA, et al., 2019).

Os estudos analisados durante a escrita da presente revisão sistemática demonstraram que o uso da Flibanserina de $50 \mathrm{mg}$ ou $100 \mathrm{mg}$, por no mínimo 24 semanas, mostrou-se efetivo no tratamento do desejo sexual hipoativo. A medicação em questão foi aprovada para o tratamento das desordens sexuais apresentadas pelas mulheres durante o período da pré-menopausa, sendo garantida a sua segurança e tolerabilidade, apesar de dois estudos apresentarem alguns efeitos colaterais comuns, como tontura, sonolência, náusea e fadiga (GOLDSTEIN IR, et al., 2017). Estes efeitos eram transitórios ou episódicos e foram de gravidade leve a moderada.

É extrema importância o adendo que grande parte dos estudos se atentam para o fato de que o desejo sexual hipoativo é uma condição multifatorial. Por esse motivo, não somente intervenções medicamentosas se mostram eficazes e suficientes para algumas mulheres, que apesar de realizarem o uso da Flibanserina, como o preconizado, ainda permanecem com uma saúde sexual insatisfatória. Além disso, a análise sobre o uso da Flibanserina deverá incluir a interpretação sobre suas principais indicações, em comparação com outros tratamentos analisados pela a literatura, levando em conta as limitações dos estudos analisados (KATZ M, et al., 2013; THORP J, et al., 2012).

\section{CONSIDERAÇÕES FINAIS}

O uso da Flibanserina para o aumento do desejo sexual em mulheres na pré-menopausa, comparado ao placebo, apresentou alta evidência de melhora do desejo sexual, quando usado por no mínimo 24 semanas, nas doses de $50 \mathrm{mg}$ ou 100mg. Dessa forma, pode-se considerar a Flibanserina uma boa opção para estimular a libido em mulheres na pré-menopausa, que sofrem com desejo sexual hipoativo, podendo também considerar o seu uso como fármaco adjuvante, pois, o desejo sexual é uma condição multifatorial. Vale ressaltar que, apesar dos avanços significativos, o tratamento de disfunção sexual feminina ainda carece de novas opções terapêuticas, tendo em vista a importância da saúde sexual e a necessidade de uma abordagem acolhedora e um tratamento multidisciplinar eficaz para o desejo sexual hipoativo.

\section{REFERÊNCIAS}

1. CLAYTON AH, et al. Flibanserin: a potential treatment for Hypoactive Sexual Desire Disorder in premenopausal women. Woman Health, 2010; 6(5): 639-653.

2. DEROGATIS LR, et al. Treatment of hypoactive sexual desire disorder in premenopausal women: efficacy of flibanserin in the VIOLET Study. Journal of Sexual Medicine, 2012; 9(4): 1074-1085.

3. FANTASIA HC. Flibanserin and Female Sexual Desire. Nursing for Women's Health, 2016; 20(5): 309-314. 
4. GELMAN F, ATRIO J. Flibanserin for hypoactive sexual desire disorder: place in therapy. Therapeutic Advances Chronic Disease, 2017; 10(8): 16-25.

5. GOLDFISCHER ER, et al. Continued efficacy and safety of flibanserin in premenopausal women with Hypoactive Sexual Desire Disorder (HSDD): results from a randomized withdrawal trial. Journal of Sexual Medicine, 2011; 8(11): 3160-3172.

6. GOLDSTEIN I, et al. Efficacy of Flibanserin by Duration of Hypoactive Sexual Desire Disorder in Premenopausal Women. Journal of Sexual Medicine, 2018; 15(6): 58-59.

7. GOLDSTEIN I, et al. Hypoactive Sexual Desire Disorder: International Society for the Study of Women's Sexual Health (ISSWSH) Expert Consensus Panel Review. Mayo Clinic Proceedings, 2017; 92(12): 114-128.

8. HOUMAN J, et al. Female Sexual Dysfunction: Is It a Treatable Disease? Current Urology Reports, 2016; 17(4): $345-$ 347.

9. JAYNE C, et al. Open-label extension study of flibanserin in women with hypoactive sexual desire disorder. Journal of Sexual Medicine, 2012; 9(12): 3180-3188.

10. JOFFE HV, et al. FDA Approval of Flibanserin--Treating Hypoactive Sexual Desire Disorder. New England Journal of Medicine, 2016; 374(15): 101-104.

11. KATZ M, et al. Efficacy of flibanserin in women with hypoactive sexual desire disorder: results from the BEGONIA trial. Journal of Sexual Medicine, 2013; 10(7): 1807-1815.

12. KRYCHMAN ML, et al. Effect of Flibanserin on Sexual Functioning: An Analysis of Female Sexual Function Index Domains. Revista Brasileira de Ginecologia e Obstetrícia, 2017; 129(5): 88S.

13. NAPPI RE. Why are there no FDA-approved treatments for female sexual dysfunction? Expert Opinion on Pharmacotherapy, 2015; 16(12): 1735-1738.

14. REVIRIEGO C. Flibanserin for female sexual dysfunction. Drugs Today (Barc), 2017; 50(12): 549-556.

15. SIMON JA, et al. Flibanserin consistently demonstrates improved sexual function in women with hypoactive desire disorder (HSDD): results from three pivotal phase 3 trials. Journal of Sexual Medicine, 2013; 10(2): 164-164.

16. SIMON JA, et al. Flibanserin for Premenopausal Hypoactive Sexual Desire Disorder: Pooled Analysis of Clinical Trials. Journal of Women's Health, 2019; 28(6): 769-777.

17. SIMON JA, et al. Flibanserin in premenopausal women with hypoactive sexual desire disorder: a responder analysis of randomized placebo-controlled studies. Journal of Sexual Medicine, 2018; 14(3): 355-357.

18. THORP J, et al. Treatment of hypoactive sexual desire disorder in premenopausal women: efficacy of flibanserin in the DAISY study. Journal of Sexual Medicine, 2012; 9(3): 793-804. 